\title{
Article
}

\section{Simple one-pot fabrication of ultra-stable core-shell superparamagnetic nanoparticles for potential application in drug delivery}

Sen, Tapas, Sheppard, Sarah J, Mercer, Tim, Eizadi-sharifabad, Maneea, Mahmoudi, Morteza and Elhissi, Adbelbary

Available at http://clok.uclan.ac.uk/7557/

Sen, Tapas ORCID: 0000-0002-0463-7485, Sheppard, Sarah J, Mercer, Tim ORCID: 0000-0002-1557-2138, Eizadi-sharifabad, Maneea, Mahmoudi, Morteza and Elhissi, Adbelbary (2012) Simple one-pot fabrication of ultrastable core-shell superparamagnetic nanoparticles for potential application in drug delivery. RSC Advances, 2 (12). pp. 5221-5228. ISSN 2046-2069

It is advisable to refer to the publisher's version if you intend to cite from the work. http://dx.doi.org/10.1039/c2ra20199b

For more information about UCLan's research in this area go to http://www.uclan.ac.uk/researchgroups/ and search for <name of research Group>.

For information about Research generally at UCLan please go to http://www.uclan.ac.uk/research/

All outputs in CLoK are protected by Intellectual Property Rights law, including Copyright law. Copyright, IPR and Moral Rights for the works on this site are retained by the individual authors and/or other copyright owners. Terms and conditions for use of this material are defined in the policies page. 


\title{
RSC Advances
}

\section{Simple one-pot fabrication of ultra-stable core-shell superparamagnetic nanoparticles for potential application in drug delivery $\dagger$}

\author{
Tapas Sen, ${ }^{* a b}$ Sarah J. Sheppard, ${ }^{a c}$ Tim Mercer, ${ }^{b d}$ Maneea Eizadi-sharifabad, ${ }^{a}$ Morteza Mahmoudi ${ }^{e f}$ and \\ Abdelbary Elhissibc
}

Received 3rd February 2012, Accepted 28th March 2012

DOI: 10.1039/c2ra20199b

\begin{abstract}
Ultrastable superparamagnetic core-shell nanoparticles of average diameter $80 \mathrm{~nm}$ have been fabricated via a simple one-pot method involving superparamagnetic iron oxide nanoparticles (SPIONs) core ( $\sim 50 \mathrm{~nm}$ in diameter) and lipid bilayer shell by high energy ultrasonication. The surface charges (zeta potentials) were measured to be between $-15 \mathrm{mV}$ and $+16 \mathrm{mV}$ depending on the batch composition. Anticancer drug mitomycin $\mathrm{C}(\mathrm{MMC})$ was loaded into four different samples of variable surface charges in aqueous solution $(\mathrm{pH}=6.8)$ and released in PBS buffer $(\mathrm{pH}=7.2)$ at room temperature. The kinetics of drug loading and releasing data indicated that the stable lipid bilayer coated SPIONs (LBCSPIONs) of nearly neutral surface exhibited the highest loading (10.9 $\mu \mathrm{g}$ of MMC/mg of materials), whereas uncoated or partially coated SPIONs of positive zeta potential exhibited the lowest loading ( 2.8 and $3.5 \mu \mathrm{g} \mathrm{MMC} / \mathrm{mg}$ of materials, respectively). The release behavior of MMC was observed to be highest (5.8 $\mathrm{gg} \mathrm{MMC} / \mathrm{mg}$ of materials) from materials of negative zeta potential compared to materials of near neutral surfaces $(3.68 \mu \mathrm{g} \mathrm{MMC} / \mathrm{mg}$ of materials). The plausible mechanism of MMC loading and releasing behavior has been explained based on the electrostatic interaction and diffusion through the lipid bilayers. To ensure biocompatibility, the interaction of the prepared SPIONs with human cervical cancer cell line (HeLa) was also investigated using an MTT (3-(4,5-dimethylthiazol-2-yl)-2,5-diphenyltetrazolium bromide) assay and ROS (reactive oxygen species) production assay and the results confirmed the supercompatibility of LBCSPIONs.
\end{abstract}

\section{Introduction}

Superparamagnetic iron oxide nanoparticles (SPIONs) have become increasingly important materials for the quick, easy, sensitive and reliable separation of specific bio-molecules and for magnetic hyperthermia agents in medical diagnosis and therapeutics. ${ }^{1-4}$ The surface properties and the inter-particle interactions are important in order to have a stable suspension of

${ }^{a}$ Centre for Materials Science, School of Forensic and Investigative Sciences, University of Central Lancashire, Preston, PRI 2HE, United Kingdom.E-mail: tsen@uclan.ac.uk; Fax:+44 1772 89 4981; Tel: +441772894371

${ }^{b}$ Institute of Nanotechnology and Bioengineering, University of Central Lancashire, Preston, PR1 2HE, United Kingdom

${ }^{c}$ School of Pharmacy and Biomedical Sciences, University of Central Lancashire, Preston, PR1 2HE, United Kingdom

${ }^{d}$ School of Computing Engineering and Physical Sciences, University of Central Lancashire, Preston, PRI $2 \mathrm{HE}$, United Kingdom

${ }^{e}$ Nanotechnology Research Center, Faculty of Pharmacy, Tehran University of Medical Sciences, Tehran, Iran

${ }^{f}$ NanoBio Interactions Laboratory, National Cell Bank, Pasteur Institute of Iran, Tehran, Iran

$\uparrow$ Electronic supplementary information (ESI) available: Standard curves for concentration of mitomycin C vs. absorbance at $365 \mathrm{~nm}$ in water (Fig. S1) and PBS buffer (Fig. S2). XRD pattern of bare SPIONs (Fig. S3), Tables S1 and S2. See DOI: 10.1039/c2ra20199b
SPIONs. One of the major problems of working on such nanoparticles is their propensity to aggregate. Surface modification of such nanoparticles in suspension raised an important question: "Does the self-assembled coating of magnetic nanoparticles cover individual particles or agglomerates?". ${ }^{5}$ A range of materials ${ }^{6-13}$ including liposomes ${ }^{14-16}$ have been used for coating SPIONs for the modification of surface charge and stability against aggregation in suspension. Sen et al. ${ }^{17}$ have reported the stability of magnetic nanoparticles using commercial dispersing agents; however, they lacked bio-compatibility for in vivo applications such as drug delivery, magnetic hyperthermia and magnetic contrasting due to chemical toxicity of the dispersing agents.

Amphiphilic phospholipid molecules in water in the form of liposomes have been well known for drug delivery for a long time $^{18-20}$ and several review papers ${ }^{21,22}$ have been published on liposomes. Drugs can be entrapped either in the inner aqueous phase or in the lipid bilayer, depending on their hydrophobicity to hydrophilicity ratio. Similarly SPIONs are also well known in the field of drug delivery ${ }^{23}$ and contrasting agents. The advantage of using SPIONs as a drug carrier is that they can be transported through the vascular system and can be concentrated at a particular point of the body with the aid of 
a magnetic field. ${ }^{24}$ Recently, we have reviewed ${ }^{25}$ the importance of SPIONs (diameter $<100 \mathrm{~nm}$ ) for chemotherapy and drug delivery as they can diffuse through the cell membrane. Size, morphology and surface charge are three important parameters for drug-loaded nanoparticles and their behaviour in the blood stream when injected intravenously. Gupta et al. ${ }^{26}$ have reported that nanoparticles ranging in diameter from 10 to $100 \mathrm{~nm}$ are most effective for drug delivery because they can evade reticuloendothelial system (RES) and hence their circulation time in blood can be prolonged. Gabizon et al. ${ }^{27}$ have reported that incorporating polyethylene glycol (PEG) to liposome bilayer results in inhibition of liposome uptake by the reticuloendothelial system and significant prolongation of liposome residence time in the blood stream. Fabrication of stable biocompatible SPIONs of diameter 10-100 nm with specific surface charge and hydrophilicity is a challenge for drug delivery due to their multifunctional properties, i.e. biocompatibility and superparamagnetism.

Magnetoliposomes are new class of nanocomposites for drug delivery $^{28-31}$ as they are biocompatible and magnetic. However, most of the reports involved larger size $(>100 \mathrm{~nm})$ liposomes where magnetite or maghemite nanoparticles have been entrapped and hence could have serious diffusional limitation. So it is always desirable to fabricate biocompatible SPIONs under $100 \mathrm{~nm}$ in diameter especially for in vivo applications. ${ }^{26}$

Mitomycin C (MMC) is a molecule that has an anticancer activity and previously been entrapped in liposomes. ${ }^{32}$ Tokunaga et $a l .{ }^{33}$ have reported the release of MMC into the blood stream through intravenous injection. Mitomycin $\mathrm{C}$ is a potent antibiotic-antineoplastic drug for ocular surgery, however it suffers due to ocular toxicity. Chetoni et al. ${ }^{34}$ have reported that liposomal preparation containing mitomycin $\mathrm{C}$ was capable of reducing the corneal healing rate and drug toxicity of a corneal lesion in a rabbit model. Zalipsky et $a l^{35}$ have reported that mitomycin-C-loaded STEALTH liposome (SL) has enhanced antitumor activity compared to pure mitomycin $\mathrm{C}$, pure doxorubicin or doxorubicin-loaded SL. Recently, Cheung et al. have reported ${ }^{36}$ the dextran-based microspheres for a loading and release study of mitomycin C. So far no reports are available on loading and release data on core-shell biocompatible SPIONs of diameter less than $100 \mathrm{~nm}$.

Herein is the first study that reports the fabrication of coreshell nanoparticles of average diameter $80 \mathrm{~nm}$ via a simple one step coating method involving SPIONs core and lipid bilayer shell by high energy ultrasonication for in vitro loading and release study of mitomycin $\mathrm{C}$ under physiological $\mathrm{pH}$. Moreover, the biocompatibility of the SPIONs were probed on human cervical cancer cell line (HeLa) using MTT (3-(4,5-dimethylthiazol-2-yl)-2,5-diphenyltetrazolium bromide) assay and ROS (reactive oxygen species) production assay.

\section{Experimental}

\subsection{Materials}

Chloroform was purchased from VWR, UK and soya phosphatidylcholine (SPC) was a gift by Lipoid, Switzerland. All other chemicals were purchased from Sigma-Aldrich and used without further purification.

\subsection{Methods}

Preparation of bare SPIONs and lipid bilayer coated SPIONs (LBCSPIONs). Bare SPIONs were synthesized following Massart method ${ }^{37}$ by co-precipitation of an aqueous solution of ferrous and ferric chloride in the presence of ammonium hydroxide with a slight modification which has been previously reported ${ }^{17}$ by Sen et al.

Multilamellar liposomes (MLVs) were prepared by dissolving $270 \mathrm{mg}$ of SPC phospholipid in $1 \mathrm{ml}$ chloroform within a $500 \mathrm{~mL}$ round bottom flask. The flask containing the phospholipid solution was attached to a rotary evaporator (Buchi Rotavapor R-114, Buchi, Switzerland) and immersed in a $35^{\circ} \mathrm{C}$ water bath. Upon evaporation of chloroform, a thin film of lipid formed on the inner wall of the flask. The flask was then flushed with nitrogen gas in order to remove chloroform residue, if any. The film was hydrated with $27 \mathrm{~mL}$ of deionized water and shaken manually for $10 \mathrm{~min}$ followed by annealing for $2 \mathrm{~h}$ at room temperature before it can be used for the preparation of LBCSPIONs. LBCSPIONs suspensions were prepared by mixing SPIONs of various amounts (see Table 1) with the MLV dispersions. The mixture was then placed under strong ultrasonic vibration (titanium horn) for 8 min using a Vibra Cell Sonicator (Sonics and Materials Inc., USA). The mixtures were repeatedly cooled using ice bath during the ultrasonication.

Powder X-ray diffraction. The X-ray diffractograms of bare SPIONs were recorded on an Inel Equinox 2000 powder diffractometer using $\mathrm{Cu}-\mathrm{K} \alpha$ radiation. The samples were prepared by drying and then grinding into a fine powder. The powder was then packed into X-ray sample holder carefully ensuring that the surface was smooth with no visible pits or cracks.

Magnetic measurements. Magnetic measurements were performed at room temperature using an in-housed vibrating sample magnetometer.

Transmission electron microscopy (TEM). Transmission electron micrographs (TEM) were recorded on a JEOL JEM2000EX (JEOL, Japan) instrument operating at an accelerating voltage $200 \mathrm{kV}$ and collected using Gatan software and digital camera. Sample suspensions were placed onto a carbon-coated copper grid using a dropping pipette and dried at RT before placing into the TEM machine.

Fourier transform infrared spectroscopy (FT-IR). All samples were characterized by FT-IR spectroscopy (SHIMADZU 8300, Shimadzu Corp. Japan) with ATR attachment. A pinch of various samples were used and the spectra were collected at RT after 15 scans in the region of 450 to $4000 \mathrm{~cm}^{-1}$.

Size and surface charge (zeta potential) analysis. Particle size analysis was performed for all samples (bare SPIONs and LBCSPIONs) using dynamic light scattering (Zetasizer Nano, Malvern Instruments, UK). The same instrument was used to analyze the zeta potential (surface charge) of the nanoparticles using Laser Doppler Velocimetry. 
Table 1 Various batches of lipid bilayers coated superparamagnetic iron oxide nanoparticles (LBCSPIONs)

\begin{tabular}{|c|c|c|c|c|}
\hline Batch No & $\begin{array}{l}\text { Concentration of } \\
\text { prefabricated bare } \\
\text { SPIONs }\left(\mathrm{mg} \mathrm{mL}^{-1}\right) \text { in } \\
\text { the final mixture }\end{array}$ & $\begin{array}{l}\text { Amount of } \\
\text { prefabricated } \\
\text { multilamellar vesicles } \\
(\mathrm{MLV}) \text { in } \mathrm{mL}\end{array}$ & $\begin{array}{l}\text { Total volume of the } \\
\text { suspension during the } \\
\text { fabrication process }\end{array}$ & $\begin{array}{l}\text { Visual observation } \\
\text { (physical stability in } \\
\text { suspension) }\end{array}$ \\
\hline SS068 & 5 & 0 & 7 & Unstable \\
\hline SS067 & 0.5 & 7 & 7 & Moderately stable \\
\hline SS063 & 2.5 & 7 & 7 & Stable \\
\hline SS069 & 5 & 7 & 7 & Stable \\
\hline SS070 & 10 & 7 & 7 & Moderately stable \\
\hline SS073 & 15 & 7 & 7 & Unstable \\
\hline SS065 & 1.25 & 2.24 & 7 & Stable \\
\hline
\end{tabular}
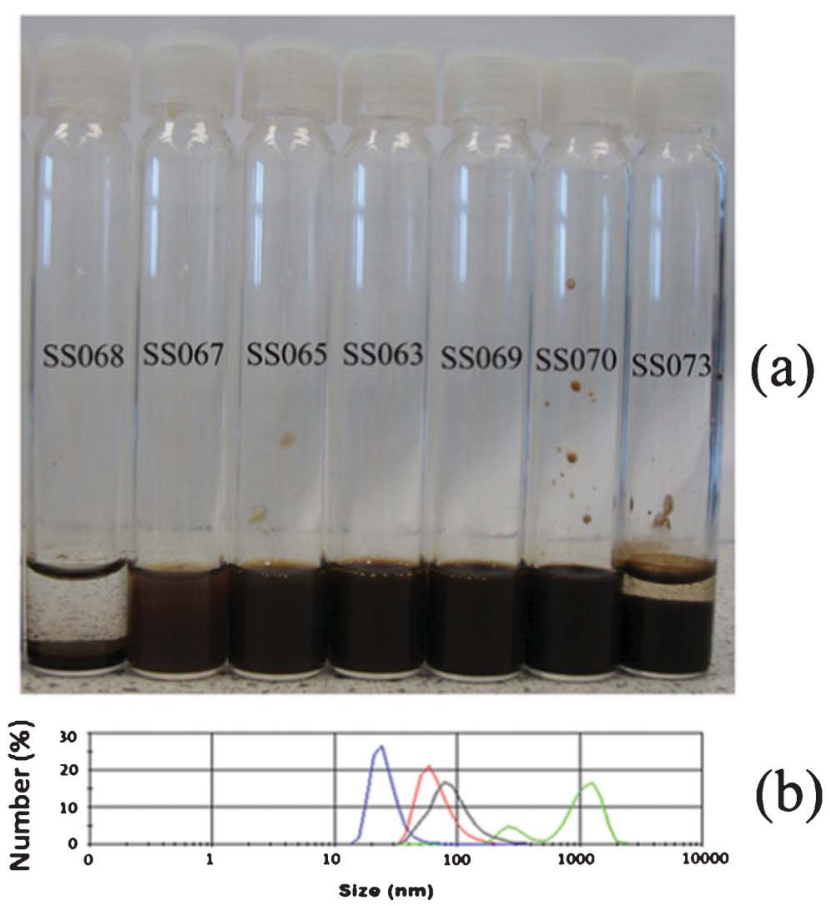

(b)

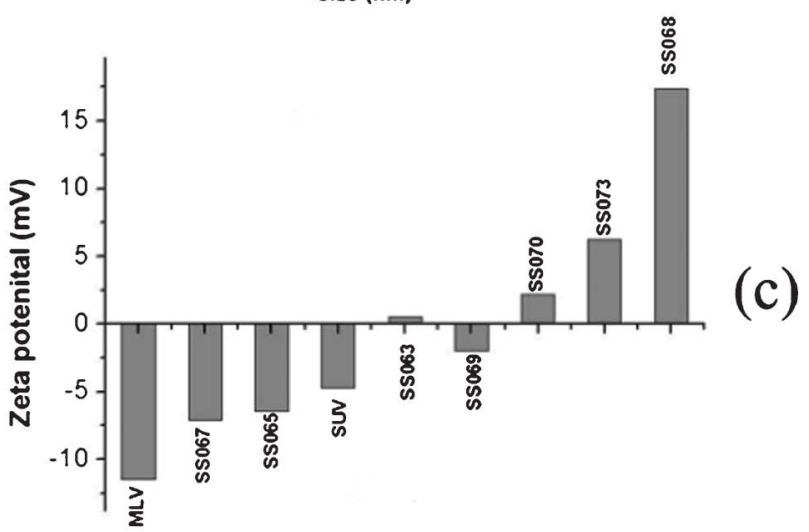

Fig. 1 (a) Photographs of various samples (b) size distribution of bare SPIONs after ultrasonication (red), MLV before (green) and after (blue) ultrasonication and LBCSPIONs SS069 (black); (c) zeta potential data of various samples.

Mitomycin C (drug) loading into the LBCSPIONs. $4 \mathrm{mg}$ of various samples (see Table $\mathrm{S} 1$ in ESI†) were placed in $1.5 \mathrm{~mL}$ Eppendorf tubes and incubated with $1 \mathrm{~mL}$ of aqueous solution of MMC having a concentration of $71 \mu \mathrm{g} \mathrm{mL} \mathrm{L}^{-1}$ at $25^{\circ} \mathrm{C}$ for up to $48 \mathrm{~h}$. The amount of MMC loaded into the nanomaterials at different time intervals were determined by measuring the UV absorption at $365 \mathrm{~nm}$ before and after the incubation with nanoparticles. The concentrations of MMC were determined by comparing the absorption $\left(\lambda_{365} \mathrm{~nm}\right)$ values with a pre-established standard curve (see Fig. S1 in ESI) of known MMC concentrations in water. MMC-loaded nanoparticles were separated from the reaction solution by centrifugation (2000 rpm for $1 \mathrm{~min}$ ) followed by magnetic separation and washed with deionised water $(\times 3,1 \mathrm{~mL})$ before being used for the release study.

Release of mitomycin C from nanomaterials in PBS buffer. $4 \mathrm{mg}$ of washed nanoparticles were treated with $1 \mathrm{~mL}$ of PBS buffer ( $\mathrm{pH}=7.2)$ at $25^{\circ} \mathrm{C}$ under stirring (end-over-end rotation) for up to $48 \mathrm{~h}$. The nanoparticles were separated in different time intervals (see Table S2 in ESI $\dagger$ ) from the solution by centrifugation (2000 rpm for $1 \mathrm{~min}$ ) and magnetic separation and the absorbance was measured at $365 \mathrm{~nm}$. The concentrations of MMC in the solution were determined by comparing the absorption $\left(\lambda_{365} \mathrm{~nm}\right)$ value with a pre-established standard curve (see Fig. S2 in ESI) of known MMC concentrations in PBS buffer.

In vitro biocompatibility assessment. Human cervical cancer cells (HeLa) from the National Cell Bank of Iran (NCBI), Pasteur Institute, were seeded on glass coverslips in 96-well plates at 10000 cells per well in $150 \mu \mathrm{L}$ of medium and incubated for $24 \mathrm{~h}$. Cells were cultured in Dulbecco's modified Eagle's medium (DMEM) supplemented with 10\% fetal bovine serum (FBS) at $37{ }^{\circ} \mathrm{C}$ in a $5 \% \mathrm{CO}_{2}$ incubator. It is well recognised that the conventional in vitro examination method may contain large errors due to the fact that nanoparticles can cause significant changes in the cell medium, such as adsorption/denaturation of proteins. ${ }^{38,39}$ In order to obtain reliable and reproducible results, the modified cytotoxicity method was employed. ${ }^{40,41}$ After the $24 \mathrm{~h}$ incubation period, $40 \mu \mathrm{L}$ of medium containing various concentrations of SPIONs (i.e. 125,250 , and $500 \mu \mathrm{g} \mathrm{ml}^{-1}$ ) was added to the wells, and cells were incubated for additional periods ranging from $12 \mathrm{~h}$. Control cells were incubated with the same culture medium without particles. All particle concentrations and controls were each seeded in ten separate wells. Cytotoxicity was assessed using the MTT (3-(4,5-dimethylthiazol-2-yl)-2,5-diphenyltetrazolium bromide) and ROS (reactive oxygen species) assay.

MTT assay. After $12 \mathrm{~h}$ incubation of nanoparticles with the cells, $100 \mu \mathrm{L}$ of MTT $\left(0.5 \mathrm{mg} \mathrm{mL}^{-1}\right)$ was added to each well. 
TEM Images
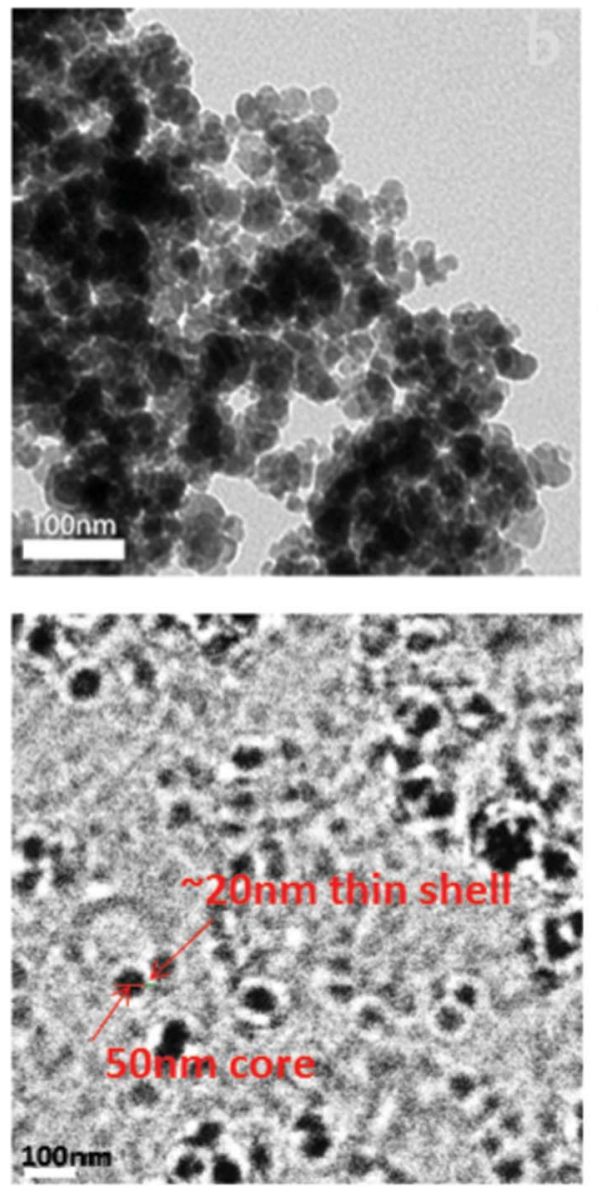

Magnetic data
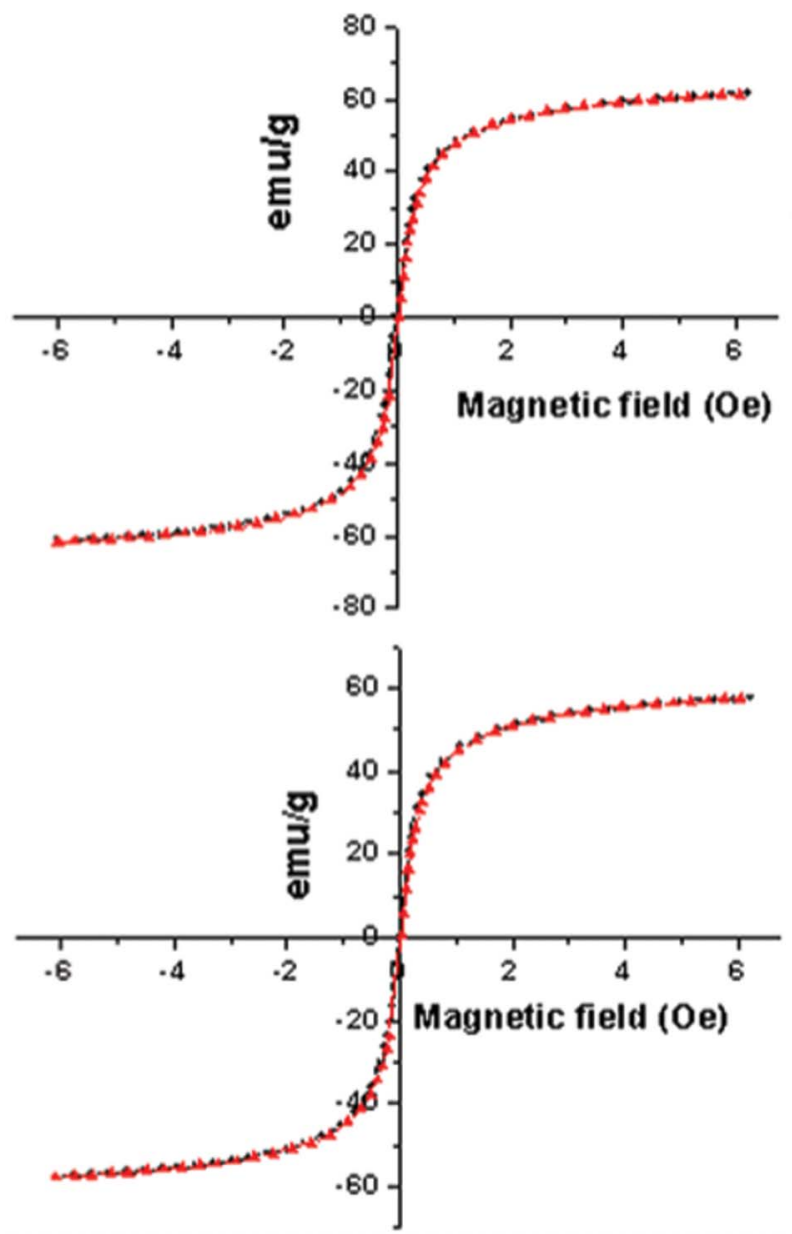

Fig. 2 TEM (left panel) of uncoated SPIONs (SS068) at top, LBCSPIONs (SS069) at the bottom; magnetic data (right panel) of bare SPIONs (SS068) at the top and LBCSPIONs (SS069) at the bottom.

Following incubation, the medium was removed and formazan crystals were solubilised by incubation for $20 \mathrm{~min}$ in $150 \mu \mathrm{L}$ of isopropyl alcohol. The absorbance of each well, which assesses viable cells, was read at $545 \mathrm{~nm}$ on a microplate reader (Stat Fax2100, Awareness, Palm City, FL).

ROS production. For the determination of intracellular ROS levels the fluorescent dye $2^{\prime}, 7^{\prime}$-dichlorodihydrofluorescein diacetate $\left(\mathrm{H}_{2} \mathrm{DCF}-\mathrm{DA}\right)$ (Invitrogen) was used. In the presence of intracellular esterases this nonpolar component is converted into the nonfluorescent polar derivative $\mathrm{H}_{2} \mathrm{DCF}$. This intermediate is membrane impermeable and rapidly oxidized to fluorescent 2',7dichlorofluorescein (DCF) by ROS. $50 \mu \mathrm{M} \mathrm{H} \mathrm{H}_{2}$ DCF-DA was added to the incubated cells-nanoparticles and the intracellular DCF fluorescence, after $30 \mathrm{~min}$, was measured with a plate reader infinite M200 (Tecan) with an excitation wavelength of $485 \mathrm{~nm}$. Emission was recorded at $535 \mathrm{~nm}$. All data were corrected for background fluorescence.

Outlier detection. The results expressed as mean (standard deviation) of all repeats. The standard deviation values are indicated as error bars in the MTT and ROS results plots. The results were statistically processed for outlier detection ${ }^{42,43}$ using a "T procedure" using MINITAB software (Minitab Inc., State College, PA). One-way analysis of variance (ANOVA) with $p<0.05$ was performed for each set of test repeats. Outlier samples have then been excluded from the corresponding assays calculations.

\section{Results and discussion}

LBCSPIONs (see Table 1) were stable in suspension (see Fig. 1a) up to the concentration of $10 \mathrm{mg} \mathrm{mL}^{-1}$ of SPIONs during the fabrication. Bare SPIONs (SS068) were unstable even after strong ultrasonication. Increasing the SPIONs concentration from $10 \mathrm{mg} \mathrm{mL}^{-1}$ to $15 \mathrm{mg} \mathrm{mL}^{-1}$ containing the same concentration of MLVs during the fabrication produced unstable suspensions (SS073).

Fig. $1 b$ represents the particle size distribution of bare SPIONs (SS068), MLVs before and after the ultrasonication and LBCSPIONs (SS069). MLVs before the ultrasonication exhibited a bimodal distribution with particle size ranging from 300 $\mathrm{nm}$ to few microns, however monomodal distribution of a size around $30 \mathrm{~nm}$ was observed after high energy ultrasonication of the MLVs. Bare SPIONs exhibited a monomodal size distribution of peak centered at around $50 \mathrm{~nm}$. LBCSPIONs (SS069) 


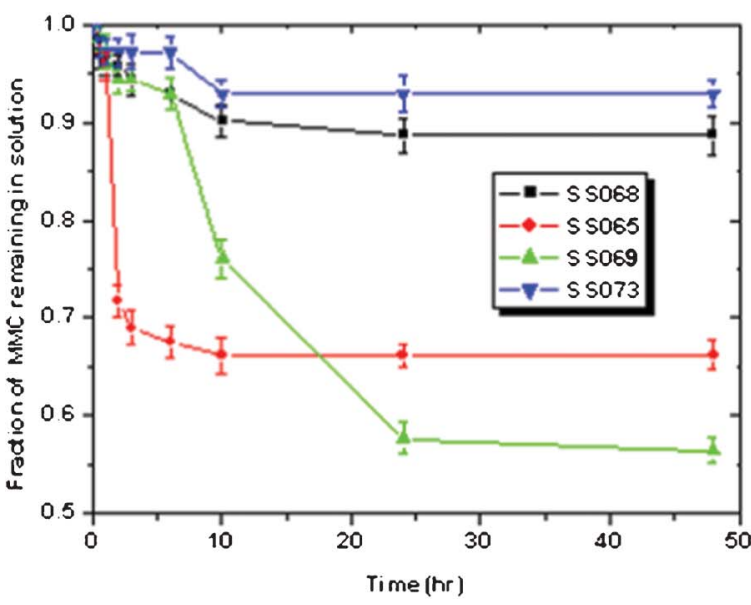

(a)

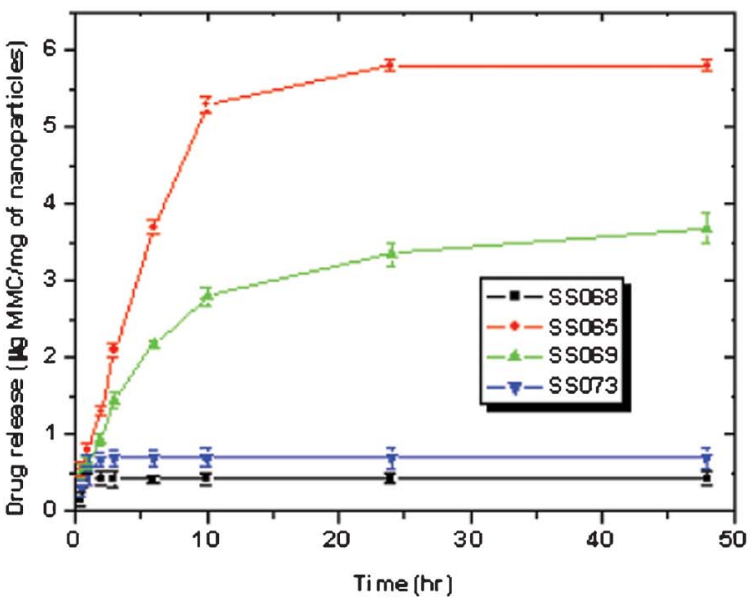

(b)

Fig. 3 Kinetics of MMC load (a) and release (b) to and from bare SPIONs and LBCSPIONs.

exhibited a monomodal size distribution of peak centered at approximately $80 \mathrm{~nm}$, indicating a clear shift in the size distribution of SPIONs after coating with the phospholipid bilayer. Fig. 1c presents the zeta potential data of SPIONs and LBCSPIONs. Bare SPIONs exhibited a positive zeta potential whereas MLVs before and after ultrasonication exhibited negative zeta potential values. Coating SPIONs with lipid bilayer decreases the zeta potential from positive to negative values depending on the concentration of SPIONs in solution during the fabrication of LBCSPIONs. Low values of zeta potential were observed on stable LBCSPIONs with optimum concentration of SPIONs in suspension (SS063, 069 and 070). This data indicates that the SPIONs were coated with phospholipid up to an optimum SPIONs concentration and the surface charge of the nanoparticles reversed by coating with lipid.

Transmission electron micrographs (see left panel of Fig. 2) exhibited that bare SPIONs were spherical in morphology of sizes ranging from $30-60 \mathrm{~nm}$ in diameter with powder X-ray diffraction peaks $(220,311,400,440)$ of fingerprint of pure magnetite $\left(\mathrm{Fe}_{3} \mathrm{O}_{4}\right)$ in the $2 \theta$ range 30 to $75^{\circ}$ (see Fig. S3 in ESI $\dagger$ ). The low intensity of the peaks is an indication of ultrasmall size of magnetite. Almost closed hysteresis loops with negligible coercivity were observed, which is indicative of the superparamagnetic nature of the iron oxide nanoparticles before and

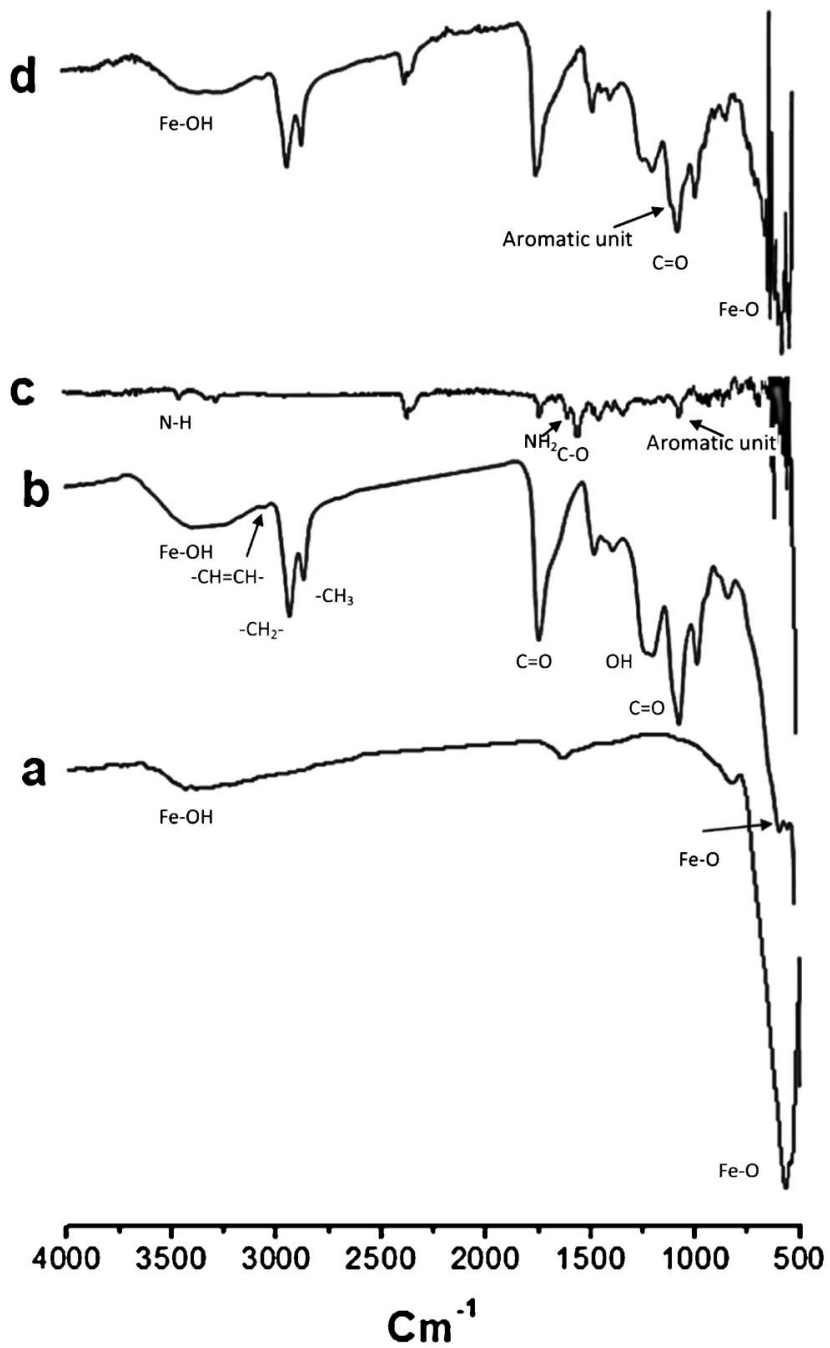

Fig. 4 FT-IR spectra of various samples: pure magnetite nanoparticles SS068 (a); bi-layer coated SS069 (b); dry mitomycin C (c); and mitomycin C loaded SS069 (d).

after coating with lipid bilayer (see right panel of Fig. 2). The coating of lipid bilayer had little effect on the saturation magnetization value $\left(\sim 60 \mathrm{emu} \mathrm{g}{ }^{-1}\right)$.

The uptake of MMC from the solution was plotted against time and presented in Fig. 3a. It was observed that the bare SPIONs (SS068) or LBCSPIONs (SS073) exhibited low uptake values $(<12 \%)$ of MMC from the solution. The highest uptake $(\sim 43 \%)$ of MMC was observed for SS069 whereas SS065 exhibited an intermediate uptake value $(\sim 32 \%)$. Saturation of MMC uptake was observed after $24 \mathrm{~h}$ of incubation for all samples except for SS065 which exhibited a near saturation of MMC uptake after around $6 \mathrm{~h}$ of incubation. SS069 exhibited MMC uptake in two steps. A low uptake value $(<10 \%)$ was observed in the first seven hours of incubation whereas the uptake value increased to saturation (42\%) at $24 \mathrm{~h}$ of incubation. The behavior of MMC uptake in four different materials can be explained based on the interaction of MMC and the nanoparticles surface due to their different surface charges (see later).

When MMC uptake values from four different samples were converted to MMC loading in $\mu \mathrm{g}$ per $\mathrm{mg}$ of nanoparticles (see Table S1 in ESI + ), it was observed that SS069 exhibited the 


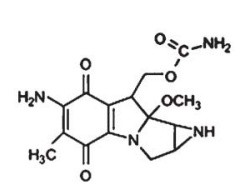

Mitomycin C

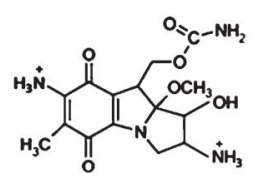

Mitomycin $\mathbf{C}$ in water (+ ve charge)

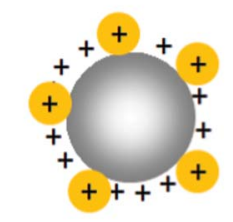

Physical adsorption SS068

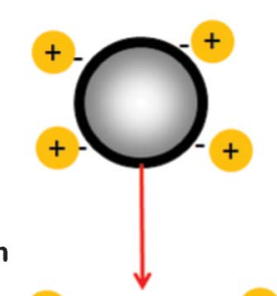

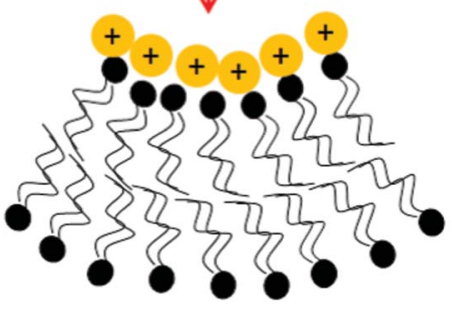

Partly lonic bonding on the surface and mostly diffusion through lipid membrane towards the iron oxide core SS069

Fig. 5 Schematic representation of the structure of MMC and the interactions between MMC and surfaces of bare SPIONs and LBCSPIONs.

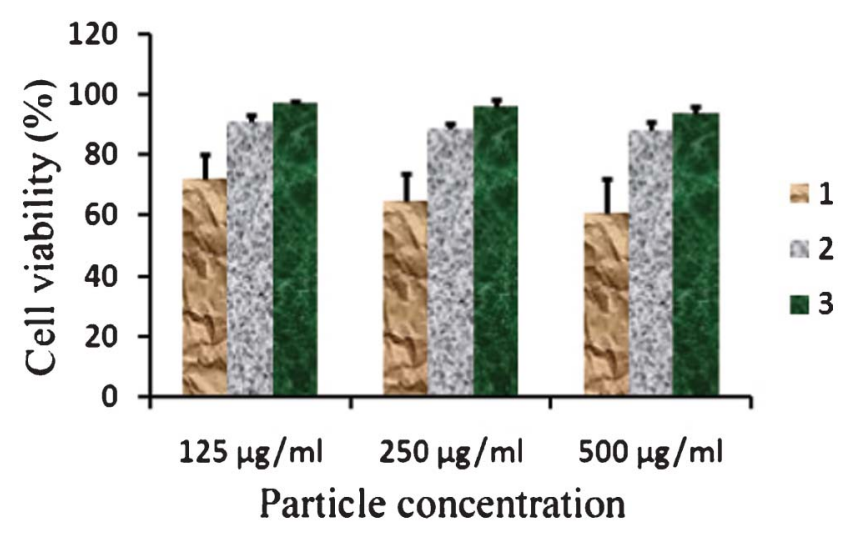

Fig. 6 MTT assay values for HeLa cells incubated with various concentrations of different SPIONs. 1: SS068, 2:SS063, 3:SS069.

highest loading $\left(\sim 11 \mu \mathrm{g} \mathrm{mg}^{-1}\right)$ at saturation point after $24 \mathrm{~h}$ of incubation whereas SS065 exhibited an intermediate value $\left(\sim 8 \mu \mathrm{g} \mathrm{mg}^{-1}\right)$. Bare SPIONs (SS068) exhibited the lowest MMC loading (2.8 $\left.\mu \mathrm{g} \mathrm{mg}^{-1}\right)$ and unstable LBCSPIONs (SS073) exhibited slightly higher loading value $\left(3.5 \mu \mathrm{g} \mathrm{mg}^{-1}\right)$.

Table S2 in ESI presents the MMC releasing data from four different materials in PBS buffer $(\mathrm{pH}=7.2)$ at $25{ }^{\circ} \mathrm{C}$. The amount of $\mathrm{MMC}$ released $(\mu \mathrm{g})$ per $\mathrm{mg}$ of nanoparticles were plotted against time (Fig. 3b). MMC release in solution quickly reached to a saturation value within two hours for both the bare SPIONs (SS068) and the unstable LBCSPIONs (SS073) and the values were calculated to be 0.43 and $0.7 \mu \mathrm{g} \mathrm{mg}^{-1}$, respectively. These values were calculated to be 15.4 and $20 \%$ of the loaded MMC in SS068 and SS073 respectively. The quick and low percentage of MMC release from SS068 and SS073 indicate these materials could be poor candidates for drug delivery application. These materials were also observed to be unstable as a suspension (see Fig. 1a) due to the aggregation of nanoparticles and, hence, can be considered unsuitable for in vivo application due to diffusional restriction through reticulo-endothelial system.

SS065 exhibited the highest $\left(\sim 6 \mu \mathrm{g} \mathrm{mg}^{-1}\right)$ MMC release and calculated to be $71 \%$ of the loaded MMC in SS065. This was achieved within $10 \mathrm{~h}$ of incubation (Fig. 3b). SS069 exhibited a gradual release of MMC up to $34 \%$ of the loaded drug after $48 \mathrm{~h}$ of incubation. The amount of MMC released from SS069 was calculated to be $3.7 \mu \mathrm{g} \mathrm{mg}^{-1}$ after $48 \mathrm{~h}$ of incubation. The highest loading of MMC in SS069 and the slow release could make this material a potential candidate for this drug delivery application in the in vivo context as they are stable against the aggregation, biocompatible and superparamagnetic in nature.

Bare SPIONs exhibited a characteristic peak (Fig. 4a) at $582 \mathrm{~cm}^{-1}$ due to $\mathrm{Fe}-\mathrm{O}$ stretching in magnetite $\left(\mathrm{Fe}_{3} \mathrm{O}_{4}\right)$, however LBCSPIONs (SS069) exhibited several additional peaks (Fig. 4b) in the region of 800 to $3500 \mathrm{~cm}^{-1}$ indicating the presence of lipid bilayer on SPIONs. Peaks at 2853 and $2922 \mathrm{~cm}^{-1}$ are the characteristics of $\mathrm{C}-\mathrm{H}$ stretching of organic tail groups. Peaks at $1725 \mathrm{~cm}^{1}$ and $1052 \mathrm{~cm}^{-1}$ are characteristics of $\mathrm{C}=\mathrm{O}$ stretching of aldehyde and ester groups respectively.

The effect of lipid bilayer shell in LBCSPIONs after MMC release has also been studied by FT-IR (Fig. 4d). Fig. 4c presents the FT-IR spectrum of pure MMC. Several peaks were observed in the region of 1000 to $3500 \mathrm{~cm}^{-1}$ with poor intensity. Peaks at 3200 to $3400 \mathrm{~cm}^{-1}$ are characteristic of MMC reported by Hou et $\mathrm{al}^{44}$ Peaks in the region of 1500 to $1700 \mathrm{~cm}^{-1}$ are characteristic of $\mathrm{C}=\mathrm{O}$ stretching and $\mathrm{N}-\mathrm{H}$ bending due to the presence of amide group. This is consistent with the structure of mitomycin C (see Fig. 5). The FT-IR spectrum of LBCSPIONs was nearly unchanged after MMC release study indicating that the lipid bilayer shell or the SPIONs core was unaffected after MMC loading and release.

Fig. 6 and 7 present the MTT assay and ROS data of HeLa cells. The results clearly show significant differences between various nanoparticles. According to the results, one can conclude that the core material (i.e. sample SS068) has lower biocompatibility value in comparison to the coated particles; the main reason is the creation of free radicals by the bare surface of SPIONs, ${ }^{45}$ which has also been confirmed by ROS results. Moreover, it was found that the produced amount of ROS in SS069-treated cells is lower than SS063 and SS068, resulting in higher compatibility of SS069 compared to uncoated SS068.

Chemical structure of $\mathrm{MMC}$ is reported ${ }^{36}$ to contain an aziridine ring which opens and forms a primary amine and hydroxyl groups by hydrolysis in water (Fig. 5). Primary amine groups later form $\mathrm{NH}_{3}{ }^{+}$ions in water and the resultant molecule can be a positively charged species. Loading and releasing MMC to and from LBCSPIONs can be explained based on the interactions between the surface of the nanoparticles and the MMC molecules. These interactions could be described as (i) electrostatic (ionic type), (ii) physical anchoring, and (iii) 


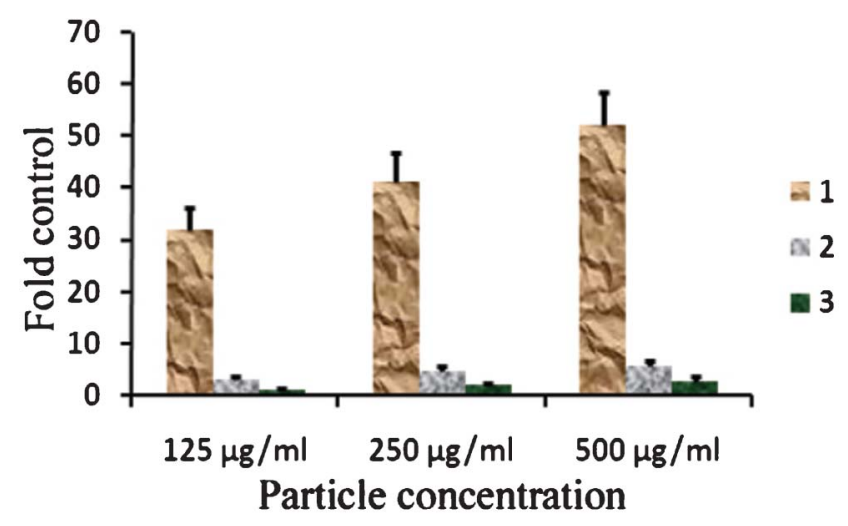

Fig. 7 Produced ROS in the HeLa cells after incubation with various concentrations of different SPIONs. 1: SS068, 2:SS063, 3:SS069.

diffusion through the lipid membrane. Electrostatic interactions could be either attractive (oppositely charged) or repulsive (similarly charged). MMC is positively charged; hence it can be easily bound to the negatively charged surface, resulting in a high loading of MMC. Similarly, positively charged surfaces can be poor for MMC loading due to the repulsive interaction. Electrostatic interactions could dominate the MMC loading behavior; however, diffusion could be a principal pathway for neutral or low surface charge. Diffusion of MMC through the lipid membrane could be a slow process hence loading and release of MMC to and from the surface could have different profile compared to electrostatic interaction. Surface of bare SPIONs (SS068) and LBCSPIONs (SS073) were measured to be positively charged hence a low MMC loading was observed (see Tables S1 and S2 in ESI $\dagger$ ) due to the repulsive interaction. A low loading value with quick saturation time could be explained based on the physical adsorption of MMC by anchoring to the surface of SS068. Release behavior of MMC from the surface was observed to be quick indicating no diffusion of $\mathrm{MMC}$ through the surface. Quick saturation times during the MMC loading (Fig. 3a) and release (Fig. 3b) to and from LBCSPIONs (SS065) with high percentage of MMC release indicate the interaction of MMC and surface of SS065 could be an electrostatic attractive type (ionic bonding). In water, positively charged MMC can be easily bound to the negatively charged surface of SS065, and can easily be released in the presence of negatively charged phosphate ions in PBS solution.

The surface of LBCSPIONs (SS069) was observed to have a weak negative charge (Fig. 2b), resulting a different pattern of MMC loading and release to and from SS069. A high value of MMC loading with a slower rate (Fig. 3a) indicates that the loading of MMC could be dominated by diffusion of MMC through the membrane due to the strong affinity of drug molecules towards the core magnetite (iron oxide surfaces). Similarly, a low (34\%) and slow MMC release profile confirms that the MMC molecules could be entrapped.

\section{Conclusions}

A series of lipid bilayer coated superparamagnetic nanoparticles (LBCSPIONs) were fabricated by a simple method using high energy ultrasonication for eventual applications in in vivo drug delivery. Loading and releasing of drug (mitomycin C) to and from the LBCSPIONs were related to the surface charges and did not have any effect on the lipid bilayer structure evidenced from FT-IR data. The super-compatibility of LBCSPIONs samples together with their suitable diameters $(<100 \mathrm{~nm})$ and slow drug release could introduce them as promising new magnetic materials for in vivo drug delivery.

\section{Acknowledgements}

SJS thank to the Learning and Development Unit (LDU) of the University of Central Lancashire for funding a summer internship (2009) to perform part of this work. We also thank to Lipoid, Switzerland, for supplying us soya phosphatidylcholine (SPC).

\section{References}

1 Q. A. Pankhurst, J. Connoll, S. K. Jones and J. Dobson, J. Phys. D: Appl. Phys., 2003, 36, R167.

2 R. Langer and D. A. Tirrell, Nature, 2004, 428, 487.

3 J. M. Perez, Nat. Nanotechnol., 2007, 2, 535.

4 T. Sen, A. Sebastianelli and I. J. Bruce, J. Am. Chem. Soc., 2006, 128, 7130.

5 R. Prozorov, T. Porozorov and A. Gedanken, Adv. Mater., 1998, 10, 1529 .

6 A. P. Philipse, M. P. B. Vanbruggen and C. Pathmamanoharan, Langmuir, 1994, 10, 92.

7 I. J. Bruce, J. Taylor, M. Todd, M. J. Davies, E. Borioni, C. Sangregorio and T. Sen, J. Magn. Magn. Mater., 2004, 284, 145.

8 T. Sen and I. J. Bruce, Microporous Mesoporous Mater., 2009, 120, 246.

9 H. L. Liu, S. P. Ko, J. H. Wu, M. H. Jung, J. H. Min, J. H. Lee, B. H. An and Y. K. Kim, J. Magn. Magn. Mater., 2007, 310, e815.

10 B. R. Jarrett, M. Frendo, J. Vogan and A. Y. Louie, Nanotechnology, 2007, 18, 035603.

11 D. K. Kim, M. Mikhaylova, F. H. Wang, J. Kehr, B. Bjelke, Y. Zhang, T. Tsakalakos and M. Muhammed, Chem. Mater., 2003, 15, 4343.

12 M. Brahler, R. Georgieva, N. Buske, A. Muller, S. Muller, J. Pinkernelle, U. Teichgraber, A. Voigt and H. Baumler, Nano Lett., 2006, 6, 2505.

13 B. Gaihre, M. S. Khil, D. R. Lee and H. Y. Kim, Int. J. Pharm., 2009, 365, 180.

14 S. Pauser, R. Reszka, S. Wagner, K. J. Wolf, H. J. Buhr and G. Berger, Anti-Cancer Drug, 1997, 12, 125.

15 P. Pradhan, J. Giri, R. Banerjee, J. Bellare and D. Bahadur, J. Magn. Magn. Mater., 2007, 311, 208.

16 M. Meincke, T. Schlorf, E. Kossel, O. Jansen, C. C. Glueer and R. Mentlein, Front. Biosci., 2008, 13, 4002.

17 T. Sen, S. Magdassi, G. Nizri and I. J. Bruce, Micro Nano Lett., 2006, 1(1), 39-42.

18 M. Mezei and V. Gulasekharam, Life Sci., 1980, 26, 1473.

19 R. L. Juliano, Trends Pharmacol. Sci., 1981, 2, 39.

20 G. Poste, C. Bucana, A. Raz, P. Bugelski, R. Kirsh and I. J. Fidler, Cancer Res., 1982, 42, 1412.

21 T. M. Allen, Drugs, 1997, 54, 8.

22 W. T. Al-Jamal and K. Kostarelos, Nanomedicine, 2007, $2,85$.

23 S. Goodwin, C. Peterson, C. Hoh and C. J. Bittner, J. Magn. Magn. Mater., 1999, 194, 132-139.

24 P. Tartaj, M. D. Morales, S. Veintemillas-Verdaguer, T. GonzalezCarreno and C. J. Serna, J. Phys. D: Appl. Phys., 2003, 36, R182.

25 M. Mahmoudi, S. Sant, B. Wang, S. Laurent and T. Sen, Adv. Drug Delivery Rev., 2011, 63, 24-46.

26 A. K. Gupta and M. Gupta, Biomaterials, 2005, 26, 3995.

27 A. A. Gabizon, H. Shmeeda and S. Zalipsky, J. Liposome Res., 2006, 16, 175.

28 M. De Cuyper, S. J. H. Soenen, K. Coenegrachts and L. Ter Beek, Anal. Biochem., 2007, 367, 266.

29 R. J. Mart, K. P. Liem and S. J. Webb, Pharm. Res., 2009, 26, 1701. 
30 S. J. H. Soenen, J. Cocquyt, L. Defour, P. Saveyn, P. Van Der Meeren and M. De Cuyper, Mater. Manuf. Processes, 2008, 23, 611.

31 S. J. H. Soenen, E. Illyes, D. Vercauteren, K. Braeckmans, Z. Majer, S. C. De Smedt and M. De Cuyper, Biomaterials, 2009, 30, 6803.

32 H. Sasaki, Y. Takakura, M. Hashida, T. Kimura and H. Sezaki, J. Pharmacobio-Dyn., 1984, 7, 120 .

33 Y. Tokunaga, T. Iwasa, Y. Tokunaga, T. Iwasa, J. Fujisaki, S. Sawai and A. Kagayama, Chem. Pharm. Bull., 1988, 36, 3557.

34 P. Chetoni, S. Burgalassi, D. Monti, M. Najarro and E. Boldrini, J. Drug Deliv. Sci. Technol., 2007, 17, 43.

35 S. Zalipsky, S. Saad, R. Kiwan, E. Ber, N. Yu and T. Minko, J. Drug Targeting, 2007, 15, 518.

36 R. Y. Cheung, Y. M. Ying, A. M. Rauth, N. Marcon and X. Y. Wu, Biomaterials, 2005, 26, 5375.

37 R. Massart, E. Dubois, V. Cabuil and E. Hasmonay, J. Magn. Magn. Mater., 1995, 149, 1.
38 M. Mahmoudi, I. Lynch, M. R. Ejtehadi, M. P. Monopoli, F. B. Bombelli and S. Laurent, Chem. Rev., 2011, 111, 5610.

39 S. Sharifi, S. Behzadi, S. Laurent, M. L. Forrest, P. Stroeve and M. Mahmoudi, Chem. Soc. Rev., 2012, 41, 2323.

40 S. Laurent, C. Burtea, C. Thirifays, U. O. Häfeli and M. Mahmoudi, PLoS One, 2012, 7, e29997, DOI: 10.1371/journal.pone.0029997.

41 M. Mahmoudi, A. Simchi, M. Imani, M. A. Shokrgozar, A. S. Milani, R. Hafeli and P. Stroeve, Colloids Surf., B, 2010, 75, 300.

42 M. Mahmoudi, A. Simchi, M. Imani, A. S. Milani and P. Stroeve, Nanotechnology, 2009, 20, 225104.

43 S. Bolton, Pharmaceutical statistics: Practical and clinical applications, 2nd edn,Marcel Dekker, New York, 1990.

44 Z. Hou, H. Wei, Q. Wang, Q. Sun, C. Zhou, C. Zhan, X. Tang and Q. Zhang, Nanoscale Res. Lett., 2009, 4, 732.

45 M. Mahmoudi, H. Hofmann, B. R. Rutishauser and A. P. Fink, Chem. Rev., 2012, 112, 2323. 